\title{
A phase II randomized trial of RAdium-223 dichloride and SABR Versus SABR for oligomEtastatic prostate caNcerS (RAVENS)
}

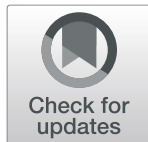

Hamza Hasan ${ }^{1 \dagger}$, Matthew P. Deek ${ }^{1 \dagger}$, Ryan Phillips ${ }^{1}$, Robert F. Hobbs ${ }^{1,2,3}$, Reem Malek, Noura Radwan', Ana P. Kiess ${ }^{1}$, Shirl Dipasquale ${ }^{1}$, James Huang ${ }^{1}$, Terry Caldwell ${ }^{1}$, Jessica Leitzel ${ }^{1}$, Danielle Wendler ${ }^{1}$, Hao Wang ${ }^{2,4}$, Elizabeth Thompson², Jonathan Powell ${ }^{2}$, Sara Dudley ${ }^{1}$, Curtiland Deville', Stephen C. Greco ${ }^{1}$, Daniel Y. Song ${ }^{1,2,5}$, Theodore L. DeWeese 1,2,5, Michael A. Gorin 2,3,5, Steven P. Rowe ${ }^{3}$, Sam Denmeade 2,5, Mark Markowski², Emmanuel S. Antonarakis ${ }^{2}$, Michael A. Carducci ${ }^{2,5}$, Mario A. Eisenberger ${ }^{2,5}$, Martin G. Pomper ${ }^{3}$, Kenneth J. Pienta ${ }^{2,5}$, Channing J. Paller ${ }^{2,5}$ and Phuoc T. Tran ${ }^{1,2,5,6^{*}}$ (D)

\section{Abstract}

Background: Metastasis directed therapy (MDT) for patients with oligometastatic disease is associated with improvements in progression free survival (PFS) and overall survival (OS) compared to systemic therapy alone. Additionally, within a prostate-cancer-specific cohort, MDT is able to forestall initiation of androgen deprivation therapy (ADT) in men with hormone-sensitive, oligometastatic prostate cancer (HSOPCa) compared to observation. While MDT appears to be safe and effective in HSOPCa, a large percentage of men will eventually have disease recurrence. Patterns of failure in HSOPCa demonstrate patients tend to have recurrence in the bone following MDT, raising the question of sub-clinically-apparent osseous disease. Radium-223 dichloride is a radiopharmaceutical with structural similarity to calcium, allowing it to be taken up by bone where it emits alpha particles, and therefore might have utility in the treatment of micrometastatic osseous disease. Therefore, the primary goal of the phase II RAVENS trial is to evaluate the efficacy of MDT + radium-223 dichloride in prolonging progression free survival in men with HSOPCa.

\footnotetext{
* Correspondence: tranp@jhmi.edu

${ }^{\dagger}$ Hamza Hasan and Matthew P. Deek contributed equally to this work.

'Department of Radiation Oncology \& Molecular Radiation Sciences, The

Sidney Kimmel Comprehensive Cancer Center, Johns Hopkins University

School of Medicine, 1550 Orleans Street, CRB2 Rm 406, Baltimore, MD 21231,

USA

2Department of Medical Oncology, The Sidney Kimmel Comprehensive

Cancer Center, Johns Hopkins University School of Medicine, Baltimore, MD,

USA

Full list of author information is available at the end of the article
}

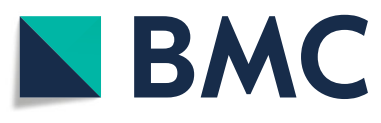

(c) The Author(s). 2020 Open Access This article is licensed under a Creative Commons Attribution 4.0 International License, which permits use, sharing, adaptation, distribution and reproduction in any medium or format, as long as you give appropriate credit to the original author(s) and the source, provide a link to the Creative Commons licence, and indicate if changes were made. The images or other third party material in this article are included in the article's Creative Commons licence, unless indicated otherwise in a credit line to the material. If material is not included in the article's Creative Commons licence and your intended use is not permitted by statutory regulation or exceeds the permitted use, you will need to obtain permission directly from the copyright holder. To view a copy of this licence, visit http://creativecommons.org/licenses/by/4.0/ The Creative Commons Public Domain Dedication waiver (http://creativecommons.org/publicdomain/zero/1.0/) applies to the data made available in this article, unless otherwise stated in a credit line to the data. 
(Continued from previous page)

Methods: Patients with HSOPCa and 3 or less metastases with at least 1 bone metastasis will be randomized 1:1 to stereotactic ablative radiation (SABR, also known as stereotactic body radiation therapy (SBRT)) alone vs SABR + radium-223 dichloride with a minimization algorithm to balance assignment by institution, primary intervention, prior hormonal therapy, and PSA doubling time. SABR is delivered in one to five fractions and patients in the SABR + radium-223 dichloride arm will receive six infusions of radium-223 dichloride at four-week intervals. The primary end point is progression free survival. The secondary clinical endpoints include toxicity and quality of life assessments, local control at 12 months, locoregional progression, time to distant progression, time to new metastasis, and duration of response.

Discussion: The RAVENS trial will be the first described phase II, non-blinded, randomized study to compare SABR +/- radium-223 dichloride in patients with HSOPCa and 3 or less metastases with at least one bone metastasis. The primary hypothesis is that SABR + radium-223 dichloride will increase median progression-free survival from 10 months in the SABR arm to 20 months in the SABR + radium-223 dichloride arm.

Trial registrations: Clinicaltrials.gov. Identifier: NCT04037358. Date of Registration: July 30, 2019. Date of First Participant Enrolled: August 9, 2019. Date of Last Approved Amendment: October 16, 2019. Protocol Version: Version 5.

Keywords: Oligometastatic, Prostate, Cancer, Stereotactic ablative radiation (SABR), Radium-223, Bone, Metastasis

\section{Background}

Cancer is currently the second leading cause of death annually in the United States, often times due to the development of metastatic disease. Systemic therapies are first line treatments in these instances and they improve survival in patients with metastatic disease. However, they are generally not considered curative for patients with solid metastatic tumors. While systemic therapies improve outcomes, that improvement can come at the expense of side effects that negatively impact patients' quality of life [1]. Thus, there have been intense efforts to develop novel therapies to improve oncologic outcomes while attempting to balance their toxicity profile.

As the experience treating metastatic disease has evolved, so too has our understanding of the natural history and biology of metastatic cancer. Metastatic disease appears to act along a spectrum that ranges from a single macroscopic metastatic deposit to widespread metastatic disease [2]. Cases with one or a few metastases (typically five or less) have been termed oligometastatic, a state first hypothesized by Hellman and Weichselbaum in the 1990s [2]. The implication of an oligometastatic state is that aggressive metastasis directed therapy (MDT) aimed at all metastatic sites can lead to long term disease control and possibly even a cure [3-6].

The feasibility of MDT has been aided by the development of stereotactic ablative radiation (SABR), a precise form of radiation therapy that allows delivery of high doses of radiation in a small number of treatment sessions. The high lesional control rates seen with SABR, in conjunction with its modest side effect profile, have since resulted in an increasing trend to treat oligometastatic lesions in an attempt to improve overall survival (OS) and progression free survival (PFS), delay initiation of systemic therapies with unfavorable toxicity profiles, and offer treatment breaks for individuals amassing toxicity from systemic therapy [7].

There is now a reasonable body of literature demonstrating the importance of local therapy in patients with oligometastatic disease. Initial evidence came from trials of non-small cell lung cancer (NSCLC) that randomized patients with de novo oligometastatic disease without progression on systemic therapy to continued maintenance systemic therapy/observation or consolidative local therapy and demonstrated improved PFS and OS with local therapy $[8,9]$. Further supporting evidence came from the recently published SABR-COMET phase II trial which randomized patients with oligorecurrent disease (and up to five metastatic lesions) with a variety of malignant histologies to receive either standard of care palliative treatments or standard of care palliative treatments plus SABR to all metastatic lesions. The results showed that the median OS in the standard of care palliative treatments arm was 28 months versus 41 months in the standard of care palliative treatments plus SABR arm [10].

Within the prostate cancer literature, numerous retrospective reports have documented the safety and feasibility of using SABR to treat oligometastatic lesions. However, the STOMP trial represented the first reported prospectively randomized trial of MDT for a prostate cancer cohort and investigated the ability of SABR to forestall initiation of androgen deprivation therapy (ADT) in men with HSOPCa with three or fewer detectable metastases. The primary endpoint was ADT-free survival, which was lengthened in men randomized to MDT versus observation (21 vs 13 months) [11, 12]. Our prospective phase II ORIOLE trial, which randomized 
men with oligometastatic disease to either MDT or observation, also reported an improvement in the primary endpoint of progression adding to the literature surrounding MDT in HSOPCa $[13,14]$.

While MDT appears to be associated with favorable outcomes in HSOPCa, a large percentage of men will eventually have disease recurrence. Patterns of failure in HSOPCa treated with MDT demonstrate that patients tend to have recurrence in an osseous site following MDT regardless of the site of the initial treated lesion [7, 15]. This raises the question of whether a significant proportion of patients have subclinical micrometastatic disease in bone sites and, if so, how this knowledge might be leveraged to improve outcomes following MDT. Radium-223 dichloride, hereafter referred to as $\left[{ }^{223} \mathrm{Ra}\right] \mathrm{RaCl}_{2}$, is a radiopharmaceutical approved by the US FDA for use in the treatment of castration-resistant prostate cancer (CRPC) with osseous metastases [16], may well be suited for this purpose. Radium-223 is effective in this regard due to its structural similarity to calcium, which causes it to be taken up in areas of bone remodeling where it then emits alpha particles [17]. Traditional beta particle-emitting radiopharmaceuticals, especially those with uptake in bone, have bone marrow as a potential organ at risk for toxicity and this was also initially a concern for $\left[{ }^{223} \mathrm{Ra}\right] \mathrm{RaCl}_{2}$. However, the short range of alpha particles relative to the size of marrow cavities (19) allows ${ }^{223} \mathrm{Ra}$ to treat osseous metastases while sparing normal tissue including bone marrow, leading to minimal side effects [18-20]. The efficacy of $\left[{ }^{223} \mathrm{Ra}\right] \mathrm{RaCl}_{2}$ in the treatment of osseous metastases in CRPC was demonstrated in a phase III randomized trial, the ALSYMPCA study, in which 921 patients were randomly assigned in a 2:1 ratio to receive six injections of $\left[{ }^{223} \mathrm{Ra}\right] \mathrm{RaCl}_{2}$ every 4 weeks or matching placebo. Those receiving $\left[{ }^{223} \mathrm{Ra}\right] \mathrm{RaCl}_{2}$ experienced a survival benefit (median, 14.9 months vs. 11.3 months) and an improvement in quality of life [16]. Therefore, the goal of our phase II RAVENS trial will be to evaluate SABR to all metastatic sites with or without the addition of $\left[{ }^{223} \mathrm{Ra}\right] \mathrm{RaCl}_{2}$ in men with HSOPCa and at least one osseous metastasis.

\section{Methods}

\section{Ethics approval}

This study was approved by the Institutional Review Board (IRB) of Johns Hopkins University (IRB00188450). The RAVENS trial is registered at the US National Institutes of Health (ClinicalTrials.gov) \#NCT04037358.

\section{Objectives}

- Primary Objective

- To assess progression-free survival of men who have HSOPCa after randomization to SABR versus SABR and $\left[{ }^{223} \mathrm{Ra}\right] \mathrm{RaCl}_{2}$.
- Secondary Objectives

- To assess the toxicity of SABR $+\left[{ }^{223} \mathrm{Ra}\right] \mathrm{RaCl}_{2}$ in patients with HSOPCa

- To determine local control at 12-months following SABR versus SABR $+\left[{ }^{223} \mathrm{Ra}\right] \mathrm{RaCl}_{2}$ in patients with HSOPCa

- To assess time to locoregional progression, time to distant progression, time to new metastasis, and duration of response following SABR versus SABR + $\left[{ }^{223} \mathrm{Ra}\right] \mathrm{RaCl}_{2}$.

- To assess ADT-free survival following SABR versus $\mathrm{SABR}+\left[{ }^{223} \mathrm{Ra}\right] \mathrm{RaCl}_{2}$.

- To assess quality of life following SABR versus $\mathrm{SABR}+\left[{ }^{223} \mathrm{Ra}\right] \mathrm{RaCl}_{2}$.

- To enumerate circulating tumor cells (CTC) using Epic Sciences' High Definition Circulating Tumor Cell (HD-CTC) platforms (Epic Sciences, San Diego, CA, USA) at Baseline and Day 181.

- To enumerate circulating tumor DNA (ctDNA) using Cancer Personalized Profiling by deep sequencing (CAPP-Seq) at Baseline, Day 91, Day 181, and Day 361.

- To quantitatively sequence T-Cell receptor (TCR) repertoires using peripheral blood monocytes and the ImmunoSEQ platform (Adaptive Biotechnologies, Seattle, WA, USA) at Baseline and Day 91.

- To evaluate immunophenotypes of peripheral blood mononuclear cells (PBMC)

- To determine the frequency of germline DNA repair mutations in the HSOPCa.

\section{Inclusion criteria}

1. Patients must have at least one, and up to three, asymptomatic metastatic tumor(s) of the bone or soft tissue (with at least one bone metastasis), diagnosed within the past 6 -months, that are $\leq 5.0$ $\mathrm{cm}$ or $<250 \mathrm{~cm}^{3}$

2. Histologic confirmation of prostate cancer (primary or metastatic tumor).

3. Patients must have had their primary tumor treated with surgery and/or radiation.

4. PSA doubling time (PSADT) $<15$ months. PSADT will be calculated using as many PSA values that are available from time of relapse (PSA > 0.2). To calculate PSADT, the Memorial Sloan Kettering Cancer Center Prostate Cancer Prediction Tool will be used, which can be found at the following web site: https://www.mskcc.org/nomograms/prostate/ psa-doubling-time.

5. Patients may have had prior systemic therapy and/ or ADT associated with treatment of their primary prostate cancer. Patients may have had ADT 
associated with salvage radiation therapy (to the primary prostate cancer or pelvis is allowed).

6. $\mathrm{PSA} \geq 0.5 \mathrm{ng} / \mathrm{mL}$ but $\leq 50 \mathrm{ng} / \mathrm{mL}$

7. Any testosterone lab within the past 6 months $>$ $50 \mathrm{ng} / \mathrm{dL}$.

8. Patients must be $\geq 18$ years of age.

9. Patients must have a life expectancy $\geq 12$ months.

10. Patients must have an Eastern Cooperative Oncology Group (ECOG) performance status $\leq 2$.

11. Patients must have normal organ and marrow function before the first administration of $\left[{ }^{223} \mathrm{Ra}\right] \mathrm{RaCl}_{2}$ defined as: the absolute neutrophil count (ANC) should be $\geq 1.5 \times 10^{9} / \mathrm{L}$, the platelet count $\geq 100 \times 10^{9} / \mathrm{L}$ and hemoglobin $\geq 10 \mathrm{~g} / \mathrm{dL}$.

12. Patients must have the ability to understand and the willingness to sign a written informed consent document.

13. Lactate dehydrogenase (LDH) level obtained within 6 months of enrollment.

\section{Exclusion criteria}

1. No more than 3 years of ADT is allowed, with the most recent ADT treatment having occurred greater than 6 months prior to enrollment.

2. Prostate-specific membrane antigen (PSMA)targeted positron emission tomography (PET)/ magnetic resonance imaging (MRI) or PSMA-PET/ computed tomography (CT) scan within the past 6 months with results that demonstrate more disease lesions than baseline $\mathrm{CT} /$ bone scan.

3. Castration-resistant prostate cancer (CRPC).

4. Spinal cord compression or impending spinal cord compression.

5. Suspected pulmonary and/or liver metastases (greater $\geq 10 \mathrm{~mm}$ in largest axis).

6. Patients receiving any other investigational therapeutic agents.

7. Patients receiving abiraterone and prednisone.

8. Patients participating in a concurrent treatment protocol.

9. Serum creatinine $>3$ times the upper limit of normal.

10. Total bilirubin $>3$ times to upper limit of normal.

11. Liver Transaminases $>5$-times the upper limit of normal.

12. Unable to lie flat during or tolerate SABR.

13. Refusal to sign informed consent.

\section{Evaluation of randomization and blinding}

This study is a multi-site, non-blinded, randomized Phase II trial in patients with oligometastatic prostate cancer with three or less metastases and at least one bone metastasis. Eligible patients will be randomized at a 1:1 ratio to one of the treatment arms: the SABR arm or the SABR $+\left[{ }^{223} \mathrm{Ra}\right] \mathrm{RaCl}_{2}$ arm (Fig. 1). The study coordinator will use an interactive web response system (IWRS) to randomize each patient. The randomization will be performed using a minimization algorithm [21] that utilizes the following stratification factors: Initial treatment (Surgery or Radiation), Prior Hormone Therapy (Yes or No), and PSADT $(<6$ months vs 6-14.9 months). The minimization algorithm uses an $85 \%$ probability of study arm assignment and has the maximum imbalance set to 4 . The randomization will not be blinded and the on-study date for protocol entry will be the day that the study subject is randomized.

\section{Interventions}

The procedures performed during the patients' pre-cycle visit will be used to determine eligibility by screening patients using the inclusion and exclusion criteria (see Tables 1 and 2).

The following will need to be completed prior to enrollment:

- PSA level will be obtained within 30 days prior to enrollment.

- Bone scan and CT of the abdomen and pelvis or MRI of the pelvis will be obtained within 3 months prior to enrollment.

- Complete blood count (CBC) with differential, LDH, serum chemistry, and testosterone will be obtained within 6 months prior to enrollment.

- Demographics, medical history, concomitant medications, physical exam, performance status, height and weight will be obtained within 30 days prior to enrollment.

The following will need to be completed on Day 1:

- Vital signs and weight.

- LDH, serum chemistry, PSA, CAPP-seq, EPIC-HDCTC, Immuno-SEQ, immunophenotyping, and rectal swab.

- $\mathrm{CBC}$ with differential (prior to $\left[{ }^{223} \mathrm{Ra}\right] \mathrm{RaCl}_{2}$ infusion, patients must meet $\mathrm{ANC} \geq 1.5 * 10^{9} / \mathrm{L}$ and platelets $\left.\geq 100 * 10^{9} / \mathrm{L}\right)$

- Brief pain inventory (short form) and common terminology criteria for adverse events (CTCAE) v4 adverse event evaluation.

The following will need to be completed between Day 1 and Day $15\left(\mathrm{SABR}+\left[{ }^{223} \mathrm{Ra}\right] \mathrm{RaCl}_{2}\right.$ arm only):

- Vital signs and weight. 


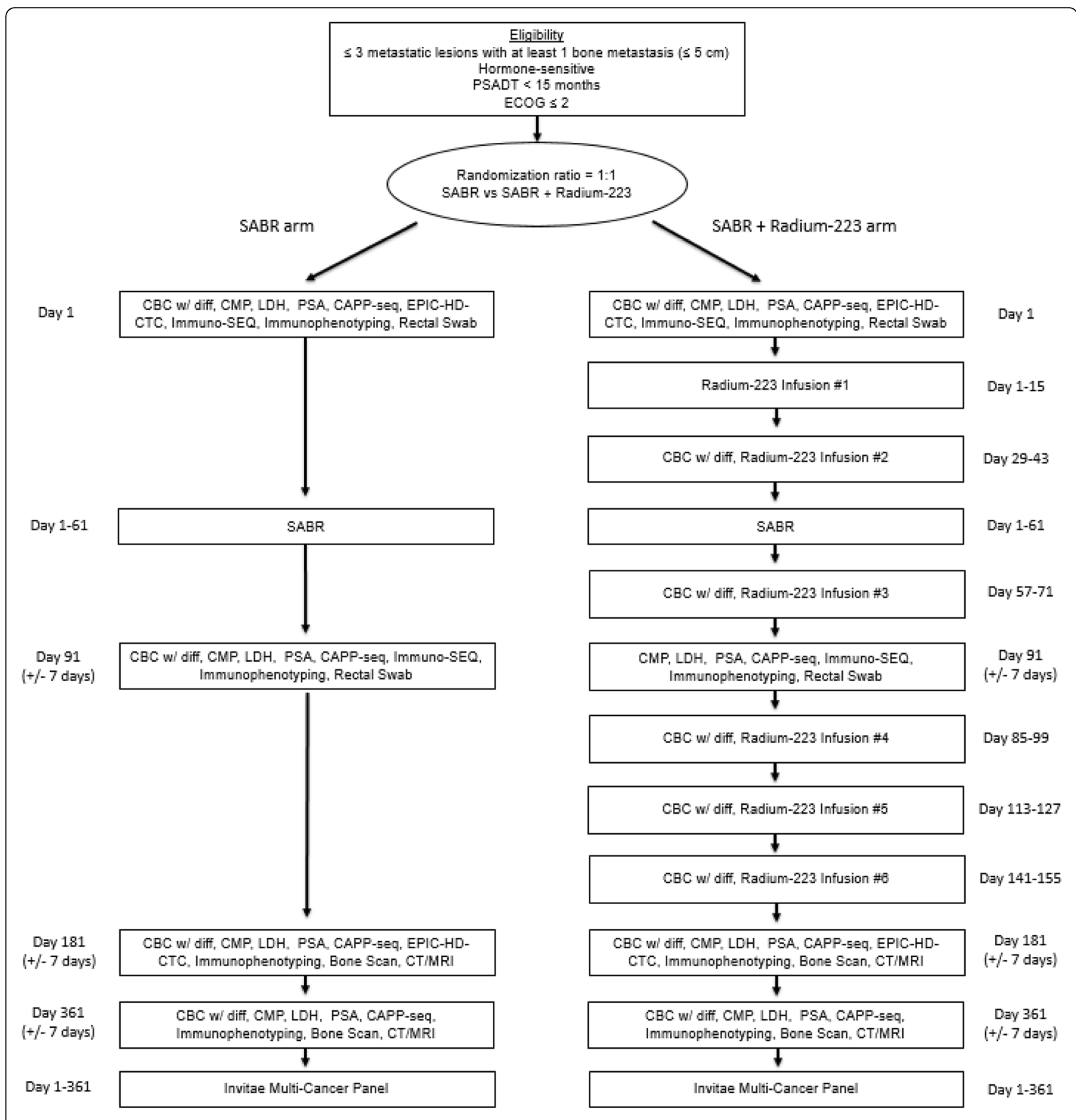

Fig. 1 Trial Schema

The following will need to be completed between Day 1 and Day 61, during the patient's SABR visit:

- Interval medical history, interval concomitant medications, limited physical exam, performance status, CTCAE v4 adverse event evaluation, and the Brief Pain Inventory (short form).

The following will need to be completed between Day 1 and Day 361:
- Invitae Multi-Cancer Panel

The following will need to be completed between Day 29 and Day 43 (SABR $+\left[{ }^{223} \mathrm{Ra}\right] \mathrm{RaCl}_{2}$ arm only)

- Vital signs and weight.

- $\mathrm{CBC}$ with differential (prior to $\left[{ }^{223} \mathrm{Ra}\right] \mathrm{RaCl}_{2}$ infusion, patients must meet ANC $\geq 1 * 10^{9} / \mathrm{L}$ and platelets $\geq$ $50 * 10^{9} / \mathrm{L}$ ) 
Table 1 Study Calendar (SABR + Xofigo arm)

\begin{tabular}{|c|c|c|c|c|c|c|c|c|c|c|c|c|c|c|c|c|c|c|}
\hline & Pre-Study & \multirow{27}{*}{ 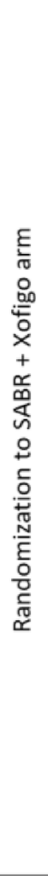 } & Day 1 & Day 15 & Day 29 & Day 43 & Day 57 & Day 61 & $D_{x y} 71$ & Day 85 & $\begin{array}{c}\text { Dey, 91 } \\
1+1+7 \text { doss }\end{array}$ & Day 99 & Day 113 & Day 127 & Day 141 & Day 155 & $\begin{array}{c}\text { Dav } 181 \\
1+7-7 \text { dass } 1\end{array}$ & $\begin{array}{c}\text { Day } 361 \\
(+1+7 \text { doyss }\end{array}$ \\
\hline ICF & $x$ & & & & & & & & & & & & & & & & & \\
\hline Demographics & $x$ & & & & & & & & & & & & & & & & & \\
\hline Medical History & $x$ & & \multicolumn{6}{|c|}{$x^{F, T}$} & & & $x^{\top}$ & & & & & & $x^{\top}$ & $x^{\top}$ \\
\hline Concomitare Medicotions & $x$ & & \multicolumn{6}{|c|}{$x^{E, T}$} & & & $x^{\top}$ & & & & & & $x^{\top}$ & $x^{\top}$ \\
\hline Physical Exam & $x$ & & \multicolumn{6}{|c|}{$x^{E, T}$} & & & $x^{\pi}$ & & & & & & $x^{\top}$ & $x^{\top}$ \\
\hline Pefformance Sravus & $x$ & & \multicolumn{6}{|c|}{$x^{F}$} & & & $x$ & & & & & & $x$ & $x$ \\
\hline Vitd Signs & $x$ & & \multicolumn{2}{|c|}{$x$} & \multicolumn{2}{|c|}{$x$} & \multicolumn{3}{|c|}{$x$} & \multicolumn{3}{|c|}{$x$} & $x$ & & \multicolumn{2}{|c|}{$x$} & & \\
\hline Height & $x$ & & & & & & & & & & & & & & & & & \\
\hline Weighte & $x$ & & \multicolumn{2}{|c|}{$x$} & \multicolumn{2}{|c|}{$x$} & \multicolumn{3}{|c|}{$x$} & \multicolumn{3}{|c|}{$x$} & $x$ & & \multicolumn{2}{|c|}{$x$} & 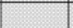 & \\
\hline $\operatorname{CBC}$ wo Oiff ${ }^{A}$ & $x^{c}$ & & $x^{\prime}$ & & \multicolumn{2}{|c|}{$x^{\prime}$} & \multicolumn{3}{|c|}{$x^{\prime}$} & \multicolumn{3}{|c|}{$x^{\prime \prime}$} & \multicolumn{2}{|c|}{$x^{\prime}$} & \multicolumn{2}{|c|}{$x^{\prime}$} & $x$ & $x$ \\
\hline $\mathrm{LOH}$ & $x^{6}$ & & $x$ & & & & & & & & $x$ & & & & & & $x$ & $x$ \\
\hline Secum Chemistry ${ }^{B}$ & $x^{c}$ & & $x$ & & & & & & & & $x$ & & & & & & $x$ & $x$ \\
\hline Testosterone & $x^{c}$ & & . & & & & & & & & $x$ & & & & & & $x$ & $x$ \\
\hline PSA & $x^{0}$ & & $x$ & & & & & & & & $x$ & & & & & & $x$ & $x$ \\
\hline Bone Scan & $x^{E}$ & & & & & & & & & & & & & & & & $x$ & $x$ \\
\hline CTMAP of Involved Site ${ }^{K}$ & $x^{E}$ & & & & & & & & & & & & & & & & $x$ & $x$ \\
\hline CAPP-seq ${ }^{\mathrm{L}}$ & & & $x^{H}$ & & & & & & & & $x^{H}$ & & & & & & $x^{H}$ & $x^{H}$ \\
\hline EAICHO-CTC ${ }^{\mathrm{M}}$ & & & $x^{H}$ & & & & & & & & & & & & & & $x^{H}$ & \\
\hline Immuno-SEQ ${ }^{N}$ & & & $x^{H}$ & & & & & & & & $x^{H}$ & & & & & & & \\
\hline Immurophenowping ${ }^{\circ}$ & & & $x^{H}$ & & & & & & & & $x^{H}$ & & & & & & $x^{H}$ & $x^{H}$ \\
\hline Invilse $^{\mathrm{P}}$ & & & & & & & & & & & 6 & & & & & & & \\
\hline Fectal Swab ${ }^{a}$ & & & $x$ & & & & & & & & $x$ & & & & & & & \\
\hline AE Evaluation & & & $x$ & & & $x^{F}$ & & & & & $x$ & & & & & & $x$ & $x$ \\
\hline Qd. Brief Pain I Imernoty & & & $x$ & & & $x^{f}$ & & & & & $x$ & & & & & & $x$ & $x$ \\
\hline $\operatorname{SABR}^{R}$ & & & & & & & & & & & & & & & & & & \\
\hline Xorigos $^{5}$ & & & $\mathrm{x}$ & & & & & $x^{\prime}$ & & & $x^{3}$ & & $x$ & & $x^{1}$ & & & \\
\hline
\end{tabular}

\begin{tabular}{|c|c|}
\hline A. & $\begin{array}{l}\text { CBC w/ Diff includes: White Blood Cell Count, Red Blood Cell Count, Hemoglobin, Hematocrit, Mean Corpuscular Volume, Mean Corpus } \mathrm{HgB}, \text { Mean Corpus HgB Conc, RBC } \\
\text { Distribution Width, Platelet Count, Neutrophils \%, Lymphocyte \%, Monocyte \%, Eosinophil \%, Basophil \%, Absolute Neutrophil Count, Absolute Lymphocyte Count, Absolute } \\
\text { Monocyte Count, Absolute Eosinphil Count, Absolute Basophil Count, Immature Granulocyte \%, and Absolute Immature Granulocyte Count. }\end{array}$ \\
\hline B. & $\begin{array}{l}\text { Serum Chemistry (CMP) includes Sodium, Potassium, Chloride, Carbon Dioxide (bicarbonate), Urea Nitrogen, Serum Creatinine, Est GFR Afr-Am(MDRD Eqn), Est GFR } \\
\text { NonAfram(MDRD Eqn), Glucose, Calcium, Total Protein, Albumin, Total Bilirubin, Alkaline Phosphatase, Aspartate Amino Trans (AST), Alanine Amino Trans (ALT), Anion Gap, } \\
\text { BUN/Creatinine Ratio, and AST/ALT ratio. }\end{array}$ \\
\hline c. & CBC w/ Diff, LDH, Serum Chemistry, and Testosterone labs must be obtained within 6 months prior to enrollment. \\
\hline D. & PSA lab must be obtained within 1 month prior to enrollment. \\
\hline E. & Pre-study Bone Scan and CT/MRI need to be obtained within 3 months prior to enrollment. \\
\hline F. & $\begin{array}{l}\text { Medical history review, concomitant medication review, physical exam, performance status assessment, adverse event evaluation, and the brief pain inventory assessment } \\
\text { should be performed once during any on treatment SABR visit. }\end{array}$ \\
\hline G. & Invitae test should be performed at any time during the study or after follow up for all enrolled patients. \\
\hline H. & Correlative study blood specimens should be drawn after standard of care labs, from the same line, to prevent epithelial cells from contaminating blood specimens. \\
\hline I. & CBC w/ Diff must be performed and analyzed prior to each Xofigo infusion. \\
\hline J. & $\begin{array}{l}\text { For patients to receive Xofigo infusion } \# 1 \text {, the following criteria must be met: } \\
\text { - ANC } \geq 1.5^{*} 10^{\circ} / \mathrm{L} \\
\text { - Platelets } \geq 100^{*} 10^{\circ} / \mathrm{L} \\
\text { - Hemoglobin } \geq 10 \mathrm{~g} / \mathrm{dL} \\
\text { For patients to receive Xofigo infusions } \mathrm{W2}-6 \text {, the following criteria must be met: } \\
\text { - ANC } \geq 11^{*} 10^{9} / \mathrm{L} \\
\text { - Platelets } \geq 50 * 10^{9} / \mathrm{L} \\
\text { If a patient fails to meet the criteria, the criteria should be re-checked every } 7-10 \text { days. If the patient does not recover to the required values within } 6-8 \text { weeks, further Xofigo } \\
\text { treatments should be discontinued. }\end{array}$ \\
\hline K. & CT/MRI should be used to measure tumar dimensions at baseline and to measure tumor dimensions and evaluate radiographic response on day 181 and on day 361. \\
\hline L. & Instructions for CAPP-Seq Sample Collection \& Processing can be found in Appendix III of the protocal. \\
\hline M. & Instructions for EPIC-HD CTC Sample Collection \& Processing can be found in Appendix IV of the protocol. \\
\hline $\mathrm{N}$. & Instructions for Immunoseq Sample Collection \& Processing can be found in Appendix V of the protocol. \\
\hline o. & Instructions for Immunophenotyping Sample Collection \& Processing can be found in Appendix VIII of the protocol. \\
\hline P. & Instructions for Invitae Sample Callection can be found in Appendix VII of the protocol. \\
\hline a & Instructions for Rectal Swab Collection for Microbiame Analysis can be found in Appendix VI of the protocol. \\
\hline R. & Instructions for SABR treatment planning can be found in section 6.3 of the protocol. \\
\hline s. & $\begin{array}{l}\text { Instructions and information for Kofigo (Radium-223) can be found in section } 6.3 \text { of the protocol. } \\
\text { Patients may sign up for the optional RAPID sub-contract SPECT imaging study. }\end{array}$ \\
\hline T. & After randomization, only interval concomitant medications, interval medical history, and limited physical exam information needs to be collected. \\
\hline U. & $\begin{array}{l}\text { Optional RAPID sub-contract SPECT imaging study: } \\
\text { SPECT scans will be performed at } 2-4 \text { hours, } 24 \text { hours }(+/ .4 \text { hours), and } 48 \text { hours }(+\%, 4 \text { hours) following Xofigo infusions } \# 1 \text { and } \# 6\end{array}$ \\
\hline
\end{tabular}


Table 2 Study Calendar (SABR arm)

\begin{tabular}{|c|c|c|c|c|c|c|c|}
\hline & Pre-Sudy & \multirow{26}{*}{ 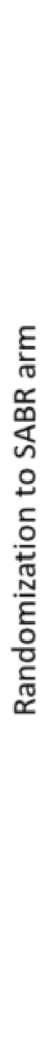 } & Das 1 & Day 61 & $\begin{array}{c}\text { Day } 91 \\
\text { (t+1-7d doys })\end{array}$ & $\begin{array}{c}\text { Day 181 } \\
{[+1-7 \text { dess })}\end{array}$ & $\begin{array}{c}\text { Day } 361 \\
{[+1-7 \text { doys }}\end{array}$ \\
\hline ICF & $x$ & & & & & & \\
\hline Demogrsphics & $x$ & & & & & & \\
\hline Medical History & $x$ & & \multicolumn{2}{|c|}{$x^{F, Q}$} & $x^{a}$ & $x^{a}$ & $x^{a}$ \\
\hline Concomitarl Medicstions & $x$ & & \multicolumn{2}{|c|}{$x^{F, Q}$} & $x^{Q}$ & $x^{a}$ & $x^{Q}$ \\
\hline Physical Exam & $x$ & & \multicolumn{2}{|c|}{$x^{F, Q}$} & $x^{a}$ & $x^{a}$ & $x^{a}$ \\
\hline Pefformance Slatus & $x$ & & \multicolumn{2}{|c|}{$x^{F}$} & $x$ & $x$ & $x$ \\
\hline Vital Signs & $x$ & & & & & & \\
\hline Heighth & $x$ & & & & & & \\
\hline Weight & $x$ & & & & & & \\
\hline$C B C w$ Diff $^{A}$ & $x^{c}$ & & $x$ & & $x$ & $x$ & $x$ \\
\hline LDH & $x^{c}$ & & $x$ & & $x$ & $x$ & $x$ \\
\hline Serum Chemistry ${ }^{B}$ & $x^{c}$ & & $x$ & & $x$ & $x$ & $x$ \\
\hline Testosterone & $x^{c}$ & & X & & $x$ & $x$ & $x$ \\
\hline PSA & $x^{D}$ & & $x$ & & $x$ & $x$ & $x$ \\
\hline Bone Scan & $x^{E}$ & & . & & & $x$ & $x$ \\
\hline CTMAFI of I Irwolved Sile ' & $x^{E}$ & & & & & $x$ & $\mathrm{x}$ \\
\hline CAPP-seq & & & $x^{H}$ & & $x^{H}$ & $x^{H}$ & $x^{H}$ \\
\hline EAICHD-CTC ${ }^{k}$ & & & $x^{H}$ & & & $x^{H}$ & \\
\hline Immuno-SEQ ${ }^{L}$ & & & $x^{H}$ & & $x^{H}$ & & \\
\hline Immunophenolyping ${ }^{\mathrm{M}}$ & & & $x^{H}$ & & $x^{H}$ & $x^{H}$ & $x^{H}$ \\
\hline Inviltes ${ }^{\mathrm{N}}$ & & & & & $x^{6}$ & & \\
\hline Fiectal Swab ${ }^{\circ}$ & & & $x$ & & $x$ & & \\
\hline AE Evaluation & & & $x$ & $\mathrm{x}$ & $x$ & $x$ & $x$ \\
\hline Dol Erief Pain Invertocy & & & $x$ & $x$ & $x$ & $x$ & $x$ \\
\hline $\operatorname{SABR}^{P}$ & & & & & & & \\
\hline
\end{tabular}

\begin{tabular}{|c|c|}
\hline A. & $\begin{array}{l}\text { CBC w/ Diff includes: White Blood Cell Count, Red Blood Cell Count, Hemoglobin, Hematocrit, Mean Corpuscular Volume, Mean } \\
\text { Corpus HgB, Mean Corpus HgB Conc, RBC Distribution Width, Platelet Count, Neutrophils \%, Lymphocyte \%, Monocyte \%, } \\
\text { Eosinophil \%, Basophil \%, Absolute Neutrophil Count, Absolute Lymphocyte Count, Absolute Monocyte Count, Absolute } \\
\text { Eosinphil Count, Absolute Basophil Count, Immature Granulocyte \%, and Absolute Immature Granulocyte Count. }\end{array}$ \\
\hline B. & $\begin{array}{l}\text { Serum Chemistry (CMP) includes Sodium, Potassium, Chloride, Carbon Dioxide (bicarbonate), Urea Nitrogen, Serum Creatinine, } \\
\text { Est GFR Afr-Am(MDRD Eqn), Est GFR NonAfrAm(MDRD Eqn), Glucose, Calcium, Total Protein, Albumin, Total Bilirubin, Alkaline } \\
\text { Phosphatase, Aspartate Amino Trans (AST), Alanine Amino Trans (ALT), Anion Gap, BUN/Creatinine Ratio, and AST/ALT ratio. }\end{array}$ \\
\hline c. & CBC w/ Diff, LDH, Serum Chemistry, and Testosterone labs must be obtained within 6 months prior to enrollment. \\
\hline D. & PSA lab must be obtained within 1 month prior to enrollment. \\
\hline E. & Pre-study Bone Scan and CT/MRII need to be obtained within 3 months prior to enrollment. \\
\hline F. & $\begin{array}{l}\text { Medical history review, concomitant medication review, physical exam, performance status assessment, adverse event } \\
\text { evaluation, and the brief pain inventory assessment should be performed once during any on treatment SABR visit. }\end{array}$ \\
\hline G. & Invitae test should be performed at any time during the study or after follow up for all enrolled patients. \\
\hline H. & $\begin{array}{l}\text { Correlative study blood specimens should be drawn after standard of care labs, from the same line, to prevent epithelial cells } \\
\text { from contaminating blood specimens. }\end{array}$ \\
\hline I. & $\begin{array}{l}\text { CT/MRI should be used to measure tumor dimensions at baseline and to measure tumor dimensions and evaluate radiographic } \\
\text { response on day } 181 \text { and on day } 361 \text {. }\end{array}$ \\
\hline J. & Instructions for CAPP-Seq Sample Collection \& Processing can be found in Appendix III of the protocol. \\
\hline K. & Instructions for EPIC-HD CTC Sample Collection \& Processing can be found in Appendix IV of the protocol. \\
\hline L. & Instructions for ImmunoSeq Sample Collection \& Processing can be found in Appendix V of the protocol. \\
\hline M. & Instructions for Immunophenotyping Sample Collection \& Processing can be found in Appendix VIII of the protocol. \\
\hline N. & Instructions for Invitae Sample Collection can be found in Appendix VII of the protocol. \\
\hline o. & Instructions for Rectal Swab Collection for Microbiome Analysis can be found in Appendix VI of the protocol. \\
\hline P. & Instructions for SABR treatment planning can be found in section 6.3 of the protocol. \\
\hline a. & $\begin{array}{l}\text { After randomization, only interval concomitant medications, interval medical history, and limited physical exam in } \\
\text { needs to be collected. }\end{array}$ \\
\hline
\end{tabular}


The following will need to be completed between Day 57 and Day 71 (SABR $+\left[{ }^{223} \mathrm{Ra}\right] \mathrm{RaCl}_{2}$ arm only)

- Vital signs and weight.

- $\mathrm{CBC}$ with differential (prior to $\left[{ }^{223} \mathrm{Ra}\right] \mathrm{RaCl}_{2}$ infusion, patients must meet $\mathrm{ANC} \geq 1 * 10^{9} / \mathrm{L}$ and platelets $\geq 50 * 10^{9} / \mathrm{L}$ )

The following will need to be completed between Day 85 and Day $99\left(\mathrm{SABR}+\left[{ }^{223} \mathrm{Ra}\right] \mathrm{RaCl}_{2}\right.$ arm only)

- Vital signs and weight.

- $\mathrm{CBC}$ with differential (prior to $\left[{ }^{223} \mathrm{Ra}\right] \mathrm{RaCl}_{2}$ infusion, patients must meet $\mathrm{ANC} \geq 1 * 10^{9} / \mathrm{L}$ and platelets $\geq 50 * 10^{9} / \mathrm{L}$ )

The following will need to be completed on Day 91:

- Interval medical history, interval concomitant medications, limited physical exam, performance status, CTCAE v4 adverse event evaluation, and the Brief Pain Inventory (short form).

- CBC with differential, LDH, serum chemistry, testosterone, PSA, CAPP-seq, Immuno-SEQ, immunophenotyping, and rectal swab.

The following will need to be completed between Day 113 and Day 127 (SABR $+\left[{ }^{223} \mathrm{Ra}\right] \mathrm{RaCl}_{2}$ arm only)

- Vital signs and weight.

- $\mathrm{CBC}$ with differential (prior to $\left[{ }^{223} \mathrm{Ra}\right] \mathrm{RaCl}_{2}$ infusion, patients must meet $\mathrm{ANC} \geq 1 * 10^{9} / \mathrm{L}$ and platelets $\geq 50 * 10^{9} / \mathrm{L}$ )

The following will need to be completed between Day 141 and Day 155 (SABR $+\left[{ }^{223} \mathrm{Ra}\right] \mathrm{RaCl}_{2}$ arm only)

- Vital signs and weight.

- $\mathrm{CBC}$ with differential (prior to $\left[{ }^{223} \mathrm{Ra}\right] \mathrm{RaCl}_{2}$ infusion, patients must meet $\mathrm{ANC} \geq 1 * 10^{9} / \mathrm{L}$ and platelets $\geq 50 * 10^{9} / \mathrm{L}$ )

The following will need to be completed on Day 181:

- Interval medical history, interval concomitant medications, limited physical exam, performance status, CTCAE v4 adverse event evaluation, and the Brief Pain Inventory (short form).

- CBC with differential, LDH, serum chemistry, testosterone, PSA, CAPP-seq, immunophenotyping, and EPIC HD-CTC.

- Bone scan and CT of the abdomen and pelvis or MRI of the pelvis..
The following will need to be completed on Day 361:

- Interval medical history, interval concomitant medications, limited physical exam, performance status, CTCAE v4 adverse event evaluation, and the Brief Pain Inventory (short form).

- CBC with differential, LDH, serum chemistry, testosterone, PSA, CAPP-seq, and immunophenotyping.

- Bone Scan and CT of the abdomen and pelvis or MRI of the pelvis.

\section{Radiation planning and dosage}

\section{- SABR Treatment Planning}

CT- and/or MRI-simulation will be performed with fabrication of a radiation therapy immobilization device (such as the Alpha Cradle) which will be custom made for each patient. The treating radiation oncologist will identify the location of the tumor. Gross tumor volume (GTV) delineation will be performed with a diagnostic radiologist on sequential axial computed tomography images. A radiosurgical treatment plan will be developed based on tumor geometry and location. The clinical tumor volume (CTV) will equal the GTV. The dose will be prescribed to the minimal isodose line that completely covers the planning target volume (PTV) which is equal to CTV plus a $3-5 \mathrm{~mm}$ margin). Adjacent normal structures including but not limited to the heart, esophagus, aorta, spinal cord, kidneys, rectum, bowel, liver, and stomach within $5 \mathrm{~cm}$ of the CTV will be identified for the purpose of limiting incidental radiation to these structures.

In addition, prior to treatment delivery, a fourdimensional cone beam CT study will be performed on individual patients to assess respiration in these patients and to determine tumor targeting accuracy for those tumors that may be subject to respiratory motion such as those in the bones of the thorax. If tumor motion is greater than $5 \mathrm{~mm}$, PTV will be expanded to account for respiration.

SABR will be delivered in 1 to 5 fractions, and the dose and fractionation schedule will depend on the size and location of the lesion and the surrounding normal tissue constraints in accordance with AAPM Task Group 101 recommendations. Typical doses include 1624 Gy in 1 fraction, 48-50 Gy in 4 fractions, and 40-60 Gy in 5 fractions.

\section{NIST standardization update}

The radioactivity quantification of ${ }^{223} \mathrm{Ra}$ in $\left[{ }^{223} \mathrm{Ra}\right] \mathrm{RaCl}_{2}$ is based on the US National Institute of Standards and Technology's (NIST) primary standardization. The US 
NIST prepares the standard reference material (SRM) using an official dial setting (primary standardization) as published [22]. The NIST SRM is used to calibrate the instruments in production and quality control for both the drug substance and drug product. Additionally, the NIST SRM is used to prepare the NIST traceable ${ }^{223} \mathrm{Ra}$ reference materials which are then sent to the end-users (e.g., nuclear medicine laboratory physicians or technicians) for dial-setting of their dose calibrators, to allow verification of the patient dose.

In 2014, NIST performed a re-assessment of the primary standardization based on preliminary information suggesting a potential discrepancy of approximately $8-10 \%$ between the published NIST primary standardization [22] and results obtained by other national metrology institutes (United Kingdom, Germany, Japan). After completion of the re-assessment, NIST reported their findings [23] and had issued a revised NIST SRM in 2015. The discrepancy in the NIST standardization was determined to be $-9.5 \%$ between activity values obtained using the old reference standard relative to the new primary standardization. Consequently, the current numerical values have been updated by approximately $+10.5 \%$.

\section{Radium-223 dichloride administration}

Dosage Forms and Strength:

$\left[{ }^{223} \mathrm{Ra}\right] \mathrm{RaCl}_{2}$ is available in single-use vials containing $6 \mathrm{~mL}$ of solution at a concentration of $1100 \mathrm{kBq} / \mathrm{mL}$ $(29.7 \mu \mathrm{Ci} / \mathrm{mL}$ ) (previously valued at $1000 \mathrm{kBq} / \mathrm{mL}$ $(27.0 \mu \mathrm{Ci} / \mathrm{mL})$ before implementation of the NIST quantification update) at the reference date with a total activity of $6600 \mathrm{kBq} /$ vial $(178 \mu \mathrm{Ci} /$ vial) (previously valued at $6000 \mathrm{kBq} /$ vial $(162 \mu \mathrm{Ci} / \mathrm{vial})$ before implementation of the NIST quantification update) at the reference date.

Before the first administration of $\left[{ }^{223} \mathrm{Ra}\right] \mathrm{RaCl}_{2}$, the ANC will be $\geq 1.5 \times 10^{9} / \mathrm{L}$, the platelet count $\geq 100 \times 10^{9} /$ $\mathrm{L}$ and hemoglobin $\geq 10 \mathrm{~g} / \mathrm{dL}$. Before subsequent administrations of $\left[{ }^{223} \mathrm{Ra}\right] \mathrm{RaCl}_{2}$, the ANC should be $\geq 1 \times 10^{9} / \mathrm{L}$ and the platelet count $\geq 50 \times 10^{9} / \mathrm{L}$. If there is no recovery to these values within 6 to 8 weeks after the last administration of $\left[{ }^{223} \mathrm{Ra}\right] \mathrm{RaCl}_{2}$, despite receiving supportive care, further treatment with $\left[{ }^{223} \mathrm{Ra}^{2} \mathrm{RaCl}_{2}\right.$ will be discontinued. Patients with evidence of compromised bone marrow reserve will be monitored closely and provided with supportive care measures when clinically indicated. $\left.{ }^{223} \mathrm{Ra}\right] \mathrm{RaCl}_{2}$ will be discontinued in patients who experience lifethreatening complications despite supportive care for bone marrow failure.

The dose regimen of $\left[{ }^{223} \mathrm{Ra}\right] \mathrm{RaCl}_{2}$ is $55 \mathrm{kBq}$ $(1.49 \mu \mathrm{Ci})$ (previously valued at $50 \mathrm{kBq}(1.35 \mu \mathrm{Ci})$ before implementation of the NIST quantification update) per $\mathrm{kg}$ body weight, given at 4-week intervals for 6 injections [24].

\section{Early stopping guidelines}

This study will monitor site-specific grade $4 / 5$ toxicity in the SABR $+\left[{ }^{223} \mathrm{Ra}\right] \mathrm{RaCl}_{2}$ arm. If it becomes evident that the proportion of grade $4 / 5$ toxicity at specific sites convincingly exceeds $20 \%$, the study will be halted for a safety consultation [14]. Patients with disease progression are individually taken off-study and adverse events are recorded for these patients.

\section{Statistical analysis \\ Response criteria}

Response criteria to treatment will be defined as follows:

- Evaluation of target lesions/PSA response [25]

- Complete Response (CR): Disappearance of all target lesions on a CT scan and bone scan and PSA $<$ preSABR PSA

- Partial Response (PR): At least 30\% decrease in the sum of the longest diameter (LD) of target lesions, taking as reference the baseline sum LD. Or a third of the lesions are negative or no change by bone scan and PSA $\leq$ pre-SABR PSA.

- Progressive Disease (PD): At least a 20\% increase in the sum of the LD of target lesions, taking as reference the smallest sum LD recorded since the treatment started or the appearance of $\geq 1$ new lesion(s) on CT or MRI. Or $\geq 1$ new lesion(s) appear by bone scan. Or $\geq 25 \%$ increase in PSA from nadir or $>50 \mathrm{ng} / \mathrm{mL}$.

- Stable Disease (SD): Neither sufficient shrinkage to qualify for PR nor sufficient increase to qualify for $\mathrm{PD}$, taking as reference the smallest sum LD since the treatment started. Or PSA $\geq$ pre-SABR PSA, but not $\geq 25 \%$ increase in PSA from nadir and $<50 \mathrm{ng} / \mathrm{mL}$.

- Evaluation of Best Overall Response The best overall response will be the best response recorded from the start of the treatment until disease progression/recurrence (taking as reference for progressive disease the smallest measurements recorded since the treatment started). Best overall response will be based on the overall response of the target lesions.

- Duration of Response Response will be defined as evidence of CR, PR, or stable disease. The duration of response will be measured from the start of treatment until the criteria for progression are met.

Duration of CR or PR: The duration of CR or PR will be recorded from the time measurement criteria are met 
for $\mathrm{CR}$ or PR (whichever is first recorded) until the first date that current or progressive disease is objectively documented (taking as reference for progressive disease the smallest measurements recorded since the treatment started).

Duration of Stable Disease: Stable disease is measured from the start of the treatment until the criteria for progression are met, taking as reference the smallest measurements recorded since the treatment started.

- Clinical Response Parameters

- Progression is a composite endpoint defined from the Prostate Cancer Working Group 2 (PCWG2) criteria for metastatic castrate resistant prostate cancer (mCRPC) [26] and our previous trials in a population of men with biochemical failure without metastases [27-29]. Progression will be defined as either: 1) a $\geq 25 \%$ increase in PSA from nadir (and by $\geq 2 \mathrm{ng} / \mathrm{mL}$ ), requiring confirmation $\geq 4$ weeks later (PCWG2 criteria); and/or, 2) clinical/radiographicprogression defined as symptomatic progression (worsening disease-related symptoms or new cancer-related complications), or radiologic progression (on CT/MRI scan: $\geq 20 \%$ enlargement in sum diameter of soft-tissue target lesions [RECIST 1.1 criteria] [25]; on bone scan: $\geq 1$ new bone lesions), initiation of ADT or death due to any cause, whichever occurs first. Death will be considered a severe adverse event here.

- Progression Free Survival (PFS) is defined as the time from starting treatment to the time of progression as defined above. Subjects who do not progress will be censored at the time of the last contact.

- ADT Free Survival (ADT-FS) is defined as the time from starting treatment to the time of initiation of palliative ADT. ADT will typically be initiated on tumor progression and/or development of new metastases. Subjects who do not start ADT will be censored at the time of the last contact.

- Time to Progression (TTP) is defined as the time from starting treatment to the time of first documented tumor progression or new lesions by CT/MRI and/or bone scan or initiation of ADT. Subjects who do not progress will be censored at the time of the last contact. In addition, death from any cause will also be censored.
- Time to New Metastasis (TTNM) is defined as the time from starting treatment to the time of a new documented tumor metastasis by CT/MRI and/or bone scan. Subjects who do not progress will be censored at the time of the last contact.

- Overall Survival (OS) is defined as the time from starting treatment until death due to any cause. For subjects who do not die, time to death will be censored at the time of last contact.

- Locoregional Control (LRC) is defined as the time from starting treatment until local and/or regional relapse is documented

\section{Statistical analysis}

- Analysis of Primary Objective This is a randomized, Phase II trial of SABR versus SABR $+\left[{ }^{223} \mathrm{Ra}\right] \mathrm{RaCl}_{2}$ in HSOPCa patients. The minimization approach [21] will be applied to ensure balanced assignment to each treatment arm by stratification factors: 1) Initial treatment with surgery vs. radiation therapy; 2) Prior hormonal therapy vs. no prior hormonal therapy; and 3) PSADT $<6$ months vs. $6-14.9$ months. Baseline PSA level is defined as that measured Day 1 following randomization.

The primary outcome of interest is PFS, defined as the time from the date of randomization to the date of disease progression or death, whichever happens earlier. For those who are alive and do not have progressive disease, PFS will be censored at the time of the last scan. The Kaplan-Meier method will be used to summarize PFS and log-rank test will be used to compare PFS between the two arms. The analysis population includes all randomized subjects based on the intent-to-treat principle. Those who are lost to follow up will be censored in the analysis.

- Analysis of Secondary Objectives

Secondary objectives will be analyzed as follows:

- For safety analysis, adverse events will be summarized by type and grade.

- Kaplan-Meier (KM) estimates will be used to summarize ADT-free survival (ADT-FS), time to locoregional progression (TTLP), time to distant progression (TTDP), time to new metastasis (TTNM) and duration of response over time. The median PFS, ADT-FS, TTLP, TTDP, TTNM, and duration of response will be reported. 
- The efficacy of SABR $+\left[{ }^{223} \mathrm{Ra}\right] \mathrm{RaCl}_{2}$ in men with HSOPCa will also be determined by measuring local control of each lesion at 12-months.

- Quality of life will be assessed using the Brief Pain Inventory form. An overall score will be calculated pre-treatment and at the time of the 2nd radiologic reassessment. The change in score will be evaluated with a paired t-test.

\section{- Sample Size}

The primary endpoint will be PFS. Data from STOMP [12] on this patient population indicate that $>50-60 \%$ would show progression as defined above within a 12-month period from SABR, and a median PFS of approximately 10 months. We hypothesize that the addition of $\left[{ }^{223} \mathrm{Ra}\right] \mathrm{RaCl}_{2}$ will be able to reduce the risk of progression by $50 \%$. A sample size using a 1:1 randomization scheme of 30 patients per arm will provide $80 \%$ power to detect an increase of median PFS from 10 months to 20 months (corresponding to hazard ratio 0.5 ) with type I error $=0.1$, using a one-sided log-rank test. The calculation assumes 18 months of accrual time with an additional follow-up of 12 months after the last patient is randomized. To account for $5 \%$ early drop out, we will randomize a total of 64 patients (32 per arm).

\section{Discussion}

Historically, aggressive local therapy has not been used in the management of patients with metastatic disease. However, with improvements in local and systemic therapy options there has recently been great interest in integrating local therapies into the management of patients with metastatic disease. This is especially the case in patients with "oligo," or few sites of metastases, who may benefit with aggressive consolidation of all macroscopic disease [7, 12, 30-42].

Several studies have now shown the clinical safety and oncologic efficacy of MDT through improvements of PFS and OS in individuals with oligometastatic disease [8-10]. Currently, the definition of oligometastatic revolves around numerical definitions due to its association with outcomes, and therefore most studies have included patients with up to 3-5 metastatic foci [43]. Little evidence exists as to whether local therapy to metastatic lesions benefits patients with higher metastatic burden. The now accruing phase III trial SABR COMET 10 (NCT03721341) is enrolling patients with 4-10 metastatic lesions with a primary end point of OS and will help answer this question [44]. If a benefit to consolidative therapy is noted with higher volume disease, $\left[{ }^{223} \mathrm{Ra}\right] \mathrm{RaCl}_{2}$ might be suited for integration into the treatment of these patients given its systemic distribution. The ALSYMPCA trial, which enrolled men with metastatic CRPC, randomized men to $\left[{ }^{223} \mathrm{Ra}\right] \mathrm{RaCl}_{2}$ or placebo and demonstrated a survival benefit for those treated with $\left[{ }^{223} \mathrm{Ra}^{2} \mathrm{RaCl}_{2}\right.$. Of these patients, $85 \%$ had $>6$ bone lesions at treatment, so $\left[{ }^{223} \mathrm{Ra}\right] \mathrm{RaCl}_{2}$ is capable of treating high volume disease [16]. Therefore, it is hoped that the results of RAVENS and SABR COMET 10 will help to inform future management in this cohort of patients.

The hormone dependent nature of prostate cancer allows for the addition of systemic therapies such as ADT to be used in the management of metastatic disease, and ADT is the first line standard of care. However, ADT is associated with side effects causing decrements in quality of life [1], so there is interest in using MDT to forestall initiation of ADT in hormone-sensitive disease. The STOMP trial, which was the first prospectively reported trial studying the efficacy of MDT in forestalling ADT initiation, showed ADT-free survival was lengthened in men randomized to MDT versus observation (21 vs 13 months) without an accompanying decrement in quality of life [12]. The RAVENS trial represents an attempt to intensify therapy in this cohort of patients while still avoiding the unfavorable toxicity profile of ADT. The short range of alpha particles emitted from ${ }^{223} \mathrm{Ra}$ results in minimal toxicity outside of occasional bone marrow suppression and thus its combination with SABR should theoretically not result in a large decrement in quality of life over SABR alone as the toxicities are orthogonal. RAVENS attempts to intensify therapy while also considering patterns of failure in oligometastatic prostate cancer following MDT, which demonstrate a trend to failure in the bone regardless of initial treatment location $[7,15] .{ }^{223} \mathrm{Ra}$ is an alpha-emitting radioisotope that is a bone-seeking calcium mimetic and selectively targets areas with increased bone turnover, especially within the microenvironment of osteoblastic or sclerotic metastases [17]. This could make $\left[{ }^{223} \mathrm{Ra}\right] \mathrm{RaCl}_{2}$ a powerful tool in forestalling disease recurrence given reported patterns of failure. Several other methods of treatment intensification are currently being investigated within the oligometastatic PCa realm, the most logical of which would be to add additional systemic therapies to MDT. Evidence suggests that the addition of a course of ADT to SABR is associated with promising outcomes. For example, a cohort of 28 men with HSOPCa at Johns Hopkins Hospital treated with a median of 4.3 months of ADT after MDT experienced a 24-month biochemical PFS of $77 \%$, with only $18 \%$ of men having restarted ADT at that time [7]. Several prospective trials are thus aiming to combine MDT with systemic agents, including combining SABR with traditional luteinizing hormone releasing hormone agonists/antagonists (NCT03940235), abiraterone (NCT03449719), ipilimumab (NCT03477864), and durvalumab (NCT03795207). Other areas of interest 
include the optimal radiation volume following nodal recurrence, being studied in the PEACE V - STORM trial, which randomizes patients to MDT and ADT +/- whole pelvis RT (NCT03569241) and the GAP6 initiative which aims to better understand molecular features of oligometastatic prostate cancer.

\section{Conclusions}

The clinical results of MDT in HSOPCa are promising but would benefit from continued novel therapeutic strategies to continue to improve outcomes. Therefore, the RAVENS trial aims to compare MDT alone to MDT plus $\left[{ }^{223} \mathrm{Ra}\right] \mathrm{RaCl}_{2}$ for patients with oligometastatic prostate cancer with 3 or less metastases and at least one bone metastasis with the primary goal of achieving improved PFS to 20 months for the patients in the SABR + $\left[{ }^{223} \mathrm{Ra}\right] \mathrm{RaCl}_{2}$.

\section{Data monitoring}

A Data Monitoring Committee is in place to monitor the trial. Data and safety monitoring oversight is conducted by the Sidney Kimmel Comprehensive Cancer Center (SKCCC) at Johns Hopkins Safety Monitoring Committee. Per the SKCCC at Johns Hopkins Safety Monitoring plan, the CRO AQ will forward summaries of all monitoring reports to the Safety Monitoring Committee for review.

\begin{abstract}
Abbreviations
[223Ra]RaCl2: Radium- 223 Dichloride; 223Ra: Radium-223; AAPM: American Association of Physicists in Medicine; ADT: Androgen Deprivation Therapy;

ADT-FS: Androgen Deprivation Therapy Free Survival; ANC: Absolute Neutrophil Count; CAPP-Seq: Cancer Personalized Profiling by Deep Sequencing; CBC: Complete Blood Count; CRPC: Castrate Resistant Prostate Cancer; CT: Computed Tomography; CTC: Circulating Tumor Cells; CTCAE: Common Terminology Criteria For Adverse Events; ctDNA: Circulating Tumor DNA; CTV: Clinical Tumor Volume; DNA: Deoxyribonucleic Acid; ECOG: Eastern Cooperative Oncology Group; GAP6: Global Action Plan 6; GTV: Gross Tumor Volume; HD: CTC High Definition Circulating Tumor Cell; HSOPCa: Hormone Sensitive Oligometastatic Prostate Cancer;

IRB: Institutional Review Board; IWRS: Interactive Web Response System; KM: Kaplan- Meier; LD: Longest Diameter; LDH: Lactate Dehydrogenase; LRC: Locoregional Control; mCRPC: Metastatic Castrate Resistant Prostate Cancer; MDT: Metastasis Directed Therapy; MRI: Magnetic Resonance Imaging; NIST: National Institute of Standards and Technology; NSCLC: NonSmall Cell Lung Cancer; OS: Overall Survival; PBMC: Peripheral Blood Mononuclear Cells; PCa: Prostate Cancer; PCWG2: Prostate Cancer Working Group 2; PD: Progressive Disease; PET: Positron Emission Tomography; PFS: Progression Free Survival; PR: Partial Response; PSA: Prostate Specific Antigen; PSADT: Prostate Specific Antigen Doubling Time; PSMA: ProstateSpecific Membrane Antigen; PTV: Planned Target Volume; RECIST: Response Evaluation Criteria in Solid Tumors; RT: Radiation Therapy; SABR: Stereotactic Ablative Radiation; SBRT: Stereotactic Body Radiation Therapy; SD: Stable Disease; SRM: Standard Reference Material; TCR: T-Cell Receptor; TTDP: Time to Distant Progression; TTLP: Time to Locoregional Progression; TTNM: Time to New Metastasis; TTP: Time to Progression; US FDA: United States Food and Drug Administration
\end{abstract}

\section{Acknowledgements}

We would like to thank the following team members for their contribution to the success of this on-going trial: Dana Kaplin, Terry Caldwell, Loretta Hollifield, and Tina Bauer.

\section{SPIRIT guidelines}

This manuscript adheres to the guidelines and methodology outlined in the SPIRIT checklist.

\section{Authors' contributions}

Study Conception: PTT. Study Design: PTT, HW. Revision of the Study: PTT, HW, HH. Drafting Manuscript: HH, MPD, RP, APK, RM, NR, [SD]2, JH, TC, JL, DW, HW, ET, JP, CD, SCG, SR, KJP, CJP, [SD]1, MM, ESA, MAC, MAG, MAE, MGP, DYS, TLD, RFH, SD, PTT. All authors have read and approved the manuscript.

\section{Funding}

BAYER HealthCare Pharmaceuticals Inc. Dr. Phuoc T. Tran is also funded by Ronald Rose \& Joan Lazar; Movember Foundation, Prostate Cancer Foundation; Commonwealth Foundation; NIH/NCI (U01CA212007, U01CA231776 and R21CA223403). The study funder had no role in the study design; collection, management, analysis, and interpretation of data; writing of the report; and the decision to submit the report for publication, including whether they will have ultimate authority over any of these activities.

\section{Availability of data and materials}

Raw data is not currently available for publication as the trial is still accruing patients and has not undergone interim analysis.

\section{Ethics approval and consent to participate}

This study will be carried out in compliance with the protocol and Good Clinical Practice, as described in: ICH Harmonized Tripartite Guidelines for Good Clinical Practice 1996; US 21 Code of Federal Regulations dealing with clinical studies (including parts 50 and 56 concerning informed consent and Institutional Review Board (IRB) regulations); and the Declaration of Helsinki, concerning medical research in humans (Recommendations Guiding Physicians in Biomedical Research Involving Human Subjects, Helsinki 1964, amended Tokyo 1975, Venice 1983, Hong Kong 1989, Somerset West 1996). The investigator agrees to adhere to the instructions and procedures described in it and thereby to adhere to the principles of Good Clinical Practice. Written informed consent is obtained from each patient before any study-specific procedure takes place. Participation in the study and date of informed consent are being documented appropriately in each patient's files. Modifications to the protocol are reported to and approved by the IRB. This study was approved by the Institutional Review Board (IRB) of Johns Hopkins University (IRB00188450).

\section{Consent for publication}

Not Applicable.

\section{Competing interests}

Robert Hobbs is a consultant for Radiopharmaceutical Imaging and Dosimetry LLC (RAPID).

\section{Author details}

'Department of Radiation Oncology \& Molecular Radiation Sciences, The Sidney Kimmel Comprehensive Cancer Center, Johns Hopkins University School of Medicine, 1550 Orleans Street, CRB2 Rm 406, Baltimore, MD 21231, USA. ${ }^{2}$ Department of Medical Oncology, The Sidney Kimmel Comprehensive Cancer Center, Johns Hopkins University School of Medicine, Baltimore, MD, USA. ${ }^{3}$ The Russell H. Morgan Department of Radiology and Radiological Science, Johns Hopkins University School of Medicine, Baltimore, MD, USA. ${ }^{4}$ Division of Biostatistics and Bioinformatics, Sidney Kimmel Comprehensive Cancer Center, Johns Hopkins University School of Medicine, Baltimore, MD, USA. ${ }^{5}$ The James Buchanan Brady Urological Institute and Department of Urology, Johns Hopkins University School of Medicine, Baltimore, MD, USA. ${ }^{6}$ Cancer Invasion and Metastasis Program, The Sidney Kimmel Comprehensive Cancer Center, Johns Hopkins University School of Medicine, Baltimore, MD, USA.

Received: 15 February 2020 Accepted: 25 May 2020

Published online: 01 June 2020

\section{References}

1. Moinpour CM, Savage MJ, Troxel A, et al. Quality of life in advanced prostate cancer: results of a randomized therapeutic trial. J Natl Cancer Inst. 1998;90:1537-44. 
2. Hellman S, Weichselbaum RR. Oligometastases. J Clin Oncol. 1995;13:8-10.

3. Bacci G, Rocca M, Salone M, et al. High grade osteosarcoma of the extremities with lung metastases at presentation: treatment with neoadjuvant chemotherapy and simultaneous resection of primary and metastatic lesions. J Surg Oncol. 2008;98:415-20.

4. Simmonds PC, Primrose JN, Colquitt JL, Garden OJ, Poston GJ, Rees M Surgical resection of hepatic metastases from colorectal cancer: a systematic review of published studies. Br J Cancer. 2006;94:982-99.

5. Fong $Y$, Cohen AM, Fortner JG, et al. Liver resection for colorectal metastases. J Clin Oncol. 1997;15:938-46.

6. Steele G Jr, Bleday R, Mayer RJ, Lindblad A, Petrelli N, Weaver D. A prospective evaluation of hepatic resection for colorectal carcinoma metastases to the liver: gastrointestinal tumor study group protocol 6584. J Clin Oncol. 1991;9:1105-12.

7. Deek MP, Yu C, Phillips $R$, et al. Radiation therapy in the definitive Management of Oligometastatic Prostate Cancer: the Johns Hopkins experience. Int J Radiat Oncol Biol Phys. 2019;105:948-56.

8. lyengar $P$, Wardak $Z$, Gerber DE, et al. Consolidative radiotherapy for limited metastatic non-small-cell lung Cancer: a phase 2 randomized clinical trial. JAMA Oncol. 2018;4:e173501.

9. Gomez DR, Blumenschein GR Jr, Lee JJ, et al. Local consolidative therapy versus maintenance therapy or observation for patients with oligometastatic non-small-cell lung cancer without progression after firstline systemic therapy: a multicentre, randomised, controlled, phase 2 study. Lancet Oncol. 2016;17:1672-82.

10. Palma DA, Olson R, Harrow S, et al. Stereotactic ablative radiotherapy versus standard of care palliative treatment in patients with oligometastatic cancers (SABR-COMET): a randomised, phase 2, open-label trial. Lancet. 2019:393:2051-8.

11. Phillips RM, Hayman J, Tran PT. STOMPing out hormone-sensitive metastases with local therapies in prostate Cancer. J Clin Oncol. 2018;36: 435-7.

12. Ost $P$, Reynders $D$, Decaestecker $K$, et al. Surveillance or metastasis-directed therapy for Oligometastatic prostate Cancer recurrence: a prospective, randomized, multicenter phase II trial. J Clin Oncol. 2018;36:446-53.

13. Radwan N, Phillips R, Ross A, et al. A phase II randomized trial of observation versus stereotactic ablative Radiatlon for OLigometastatic prostate CancEr (ORIOLE). BMC Cancer. 2017;17:453.

14. Phillips R, Shi WY, Deek M, Radwan N, Lim SJ, Antonarakis ES, Rowe SP, Ross AE, Gorin MA, Deville C, Greco SC, Wang H, Denmeade SR, Paller CJ, Dipasquale S, DeWeese TL, Song DY, Wang H, Carducci MA, Pienta KJ, Pomper MG, Dicker AP, Eisenberger MA, Alizadeh AA, Diehn M, Tran PT. Outcomes of observation vs stereotactic ablative radiation for Oligometastatic prostate Cancer: the ORIOLE phase 2 randomized clinical trial. JAMA Oncol. 2020;6(5):650-9. PMID: 32215577

15. Soldatov $A$, von Klot CAJ, Walacides $D$, et al. Patterns of progression after (68) Ga-PSMA-ligand PET/CT-guided radiation therapy for recurrent prostate Cancer. Int J Radiat Oncol Biol Phys. 2019;103:95-104.

16. Parker C, Nilsson S, Heinrich D, et al. Alpha emitter radium-223 and survival in metastatic prostate cancer. N Engl J Med. 2013;369:213-23.

17. Deshayes E, Roumiguie M, Thibault C, et al. Radium 223 dichloride for prostate cancer treatment. Drug Des Devel Ther. 2017:11:2643-51.

18. Nilsson S, Franzen L, Parker C, et al. Bone-targeted radium-223 in symptomatic, hormone-refractory prostate cancer: a randomised, multicentre, placebo-controlled phase II study. Lancet Oncol. 2007;8:587-94.

19. Nilsson S, Larsen RH, Fossa SD, et al. First clinical experience with alphaemitting radium-223 in the treatment of skeletal metastases. Clin Cancer Res. 2005:11:4451-9.

20. Hobbs RF, Song H, Watchman CJ, et al. A bone marrow toxicity model for (2)(2)(3) Ra alpha-emitter radiopharmaceutical therapy. Phys Med Biol. 2012; 57:3207-22.

21. Vickers AJ. How to randomize. J Soc Integr Oncol. 2006;4:194-8.

22. Cessna JT, Zimmerman BE. Standardization of radium-223 by liquid scintillation counting. Appl Radiat Isot. 2010;68:1523-8.

23. Zimmerman BE, Bergeron DE, Cessna JT, Fitzgerald R, Pibida L. Revision of the NIST standard for (223) Ra: new measurements and review of 2008 data. J Res Natl Inst Stand Technol. 2015;120:37-57.

24. Xofigo (radium Ra 223 dichloride) Injection [package insert]. Wayne (NJ): Bayer HealthCare Pharmaceuticals Inc. 2013. http://labeling.bayerhealthcare. com/html/products/pi/Xofigo_PI.pdf.
25. Eisenhauer EA, Therasse $P$, Bogaerts J, et al. New response evaluation criteria in solid tumours: revised RECIST guideline (version 1.1). Eur J Cancer. 2009; 45:228-47.

26. Scher HI, Halabi S, Tannock I, et al. Design and end points of clinical trials for patients with progressive prostate cancer and castrate levels of testosterone: recommendations of the prostate Cancer clinical trials working group. J Clin Oncol. 2008;26:1148-59.

27. Antonarakis ES, Feng Z, Trock BJ, et al. The natural history of metastatic progression in men with prostate-specific antigen recurrence after radical prostatectomy: long-term follow-up. BJU Int. 2012;109:32-9.

28. Keizman D, Zahurak $M$, Sinibaldi $V$, et al. Lenalidomide in nonmetastatic biochemically relapsed prostate cancer: results of a phase $1 /$ II doubleblinded, randomized study. Clin Cancer Res. 2010;16:5269-76.

29. Pound CR, Partin AW, Eisenberger MA, Chan DW, Pearson JD, Walsh PC. Natural history of progression after PSA elevation following radical prostatectomy. JAMA. 1999;281:1591-7.

30. Kneebone A, Hruby G, Ainsworth $\mathrm{H}$, et al. Stereotactic body radiotherapy for Oligometastatic prostate Cancer detected via prostate-specific membrane antigen positron emission tomography. Eur Urol Oncol. 2018;1:531-7.

31. Siva S, Bressel M, Murphy DG, et al. Stereotactic Abative body radiotherapy (SABR) for Oligometastatic prostate Cancer: a prospective clinical trial. Eur Urol. 2018;74:455-62.

32. Moyer $\mathrm{CL}$, Phillips R, Deek MP, et al. Stereotactic ablative radiation therapy for oligometastatic prostate cancer delays time-to-next systemic treatment. World J Urol. 2019;37:2623-9.

33. De Bleser E, Tran PT, Ost P. Radiotherapy as metastasis-directed therapy for oligometastatic prostate cancer. Curr Opin Urol. 2017;27:587-95.

34. Muldermans JL, Romak LB, Kwon ED, Park SS, Olivier KR. Stereotactic body radiation therapy for Oligometastatic prostate Cancer. Int J Radiat Oncol Bio Phys. 2016;95:696-702.

35. Ost P, Jereczek-Fossa BA, As NV, et al. Progression-free survival following stereotactic body radiotherapy for Oligometastatic prostate Cancer treatment-naive recurrence: a multi-institutional analysis. Eur Urol. 2016;69: 9-12.

36. Decaestecker K, De Meerleer G, Lambert B, et al. Repeated stereotactic body radiotherapy for oligometastatic prostate cancer recurrence. Radiat Oncol. 2014;9:135.

37. Ahmed KA, Barney BM, Davis BJ, Park SS, Kwon ED, Olivier KR. Stereotactic body radiation therapy in the treatment of oligometastatic prostate cancer. Front Oncol. 2012;2:215

38. Loi M, Di Cataldo V, Francolini G, et al. Single-fraction stereotactic body radiotherapy for Oligometastatic lymph node relapse in prostate Cancer. Oncol Res Treat. 2018;41:703-5.

39. Markowski MC, Imus P, Wright JL, Schottenstein D, Paller CJ. Long-term control of Oligometastatic prostate Cancer after stereotactic body radiotherapy in the absence of androgen deprivation therapy: a case report. Clin Genitourin Cancer. 2017;15:e839-e42.

40. Ingrosso G, Trippa F, Maranzano E, et al. Stereotactic body radiotherapy in oligometastatic prostate cancer patients with isolated lymph nodes involvement: a two-institution experience. World J Urol. 2017:35:45-9.

41. Pasqualetti F, Panichi M, Sainato A, et al. [(18) F] Choline PET/CT and stereotactic body radiotherapy on treatment decision making of oligometastatic prostate cancer patients: preliminary results. Radiat Oncol. 2016;11:9.

42. Schick U, Jorcano $S$, Nouet $P$, et al. Androgen deprivation and high-dose radiotherapy for oligometastatic prostate cancer patients with less than five regional and/or distant metastases. Acta Oncol. 2013;52:1622-8.

43. Tosoian JJ, Gorin MA, Ross AE, Pienta KJ, Tran PT, Schaeffer EM. Oligometastatic prostate cancer: definitions, clinical outcomes, and treatment considerations. Nat Rev Urol. 2017:14:15-25.

44. Palma DA, Olson R, Harrow S, et al. Stereotactic ablative radiotherapy for the comprehensive treatment of 4-10 oligometastatic tumors (SABR-COMET-10): study protocol for a randomized phase III trial. BMC Cancer. 2019;19:816.

\section{Publisher's Note}

Springer Nature remains neutral with regard to jurisdictional claims in published maps and institutional affiliations. 